\title{
Procalcitonin and MR-proAdrenomedullin combination in the etiological diagnosis and prognosis of sepsis and septic shock
}

\author{
Silvia Spoto ${ }^{\mathrm{a}}$, Marta Fogolari ${ }^{\mathrm{b}}$, Lucia De Florio ${ }^{\mathrm{b}}$, Marilena Minieri ${ }^{\mathrm{c}, \mathrm{d}}$, Giuseppe Vicino ${ }^{\mathrm{d}}$, \\ Jacopo Legramante $^{\mathrm{e}, \mathrm{f}}$, Maria Stella Lia ${ }^{\mathrm{d}}$, Alessandro Terrinoni ${ }^{\mathrm{c}}$, Damiano Caputo ${ }^{\mathrm{g}}$, \\ Sebastiano Costantino ${ }^{\text {a }}$, Sergio Bernardini ${ }^{\mathrm{c}, \mathrm{d}}$, Massimo Ciccozzi ${ }^{\mathrm{h}}$, Silvia Angeletti ${ }^{\mathrm{b}, *}$ \\ ${ }^{a}$ Internal Medicine Department, University Campus Bio-Medico of Rome, Italy \\ ${ }^{\mathrm{b}}$ Unit of Clinical Laboratory Science, University Campus Bio-Medico of Rome, Italy \\ ${ }^{\mathrm{c}}$ Department of Experimental Medicine, University of Tor Vergata of Rome, Italy \\ ${ }^{\mathrm{d}}$ Department of Laboratory Medicine, Policlinico Tor Vergata of Rome, Italy \\ ${ }^{\mathrm{e}}$ Emergency Department, Policlinico Tor Vergata, Roma, Italy \\ ${ }_{\mathrm{f}}^{\mathrm{f}}$ Department of Medical Systems, Università di Tor Vergata, Roma, Italy \\ ${ }^{\mathrm{g}}$ Department of Surgery, University Campus Bio-Medico of Rome, Rome, Italy \\ ${ }^{\mathrm{h}}$ Unit of Medical Statistics and Molecular Epidemiology, University Campus Bio-Medico of Rome, Rome, Italy
}

\section{A R T I C L E I N F O}

\section{Keywords:}

PCT

MR-proADM

Sepsis

Prognosis

Etiology

\begin{abstract}
A B S T R A C T
Procalcitonin and Mid-regional pro Adrenomedullin have been proposed for sepsis diagnosis, antibiotic therapy guidance and prognosis. A retrospective analysis of PCT and MR-proADM on 571 consecutive patients with sepsis diagnosis was performed. Median values were compared using the non-parametric Mann-Whitney's test. Receiver operating characteristic analysis was performed to define cutoff points for sepsis diagnosis. Pretest odds, posttest odds, and posttest probability have been calculated. Data were analyzed using Med-Calc 11.6.1.0 software. PCT resulted excellent in gram-negative, but less performant in gram-positive and fungal etiologies. MR-proADM values resulted homogenously distributed within the different microbial classes and increased significantly in septic shock. PCT highest PPV value was found to distinguish gram-negative from fungal sepsis and septic shock ( $>3.57 \mathrm{ng} / \mathrm{mL}$, PPV 0.96 and > 8.77 ng/mL, PPV 0.96, respectively). Good diagnostic accuracy was evidenced to discriminate gram-negative from gram-positive septic shock ( $>3.88 \mathrm{ng} / \mathrm{mL} \mathrm{PPV} 0.89$ ). Lower diagnostic accuracy was evidenced to discriminate gram-negative and gram-positive sepsis ( $>0.80 \mathrm{ng} /$ $\mathrm{mL}, \mathrm{PPV} 0.78$ ) and gram-positive from fungal septic shock ( $>1.74 \mathrm{ng} / \mathrm{mL}$ PPV 0.75). The lowest PCT PPV (0.28) was found in gram-positive and fungal sepsis distinction. MR-proADM discriminating cut-offs were homogeneously distributed in Gram-negative and Gram-positive sepsis and were higher in septic shock, but not influenced by pathogen etiologies. MR-proADM cut-off values $>3.39 \mathrm{nmol} / \mathrm{L}$ in sepsis and $>4.33 \mathrm{nmol} / \mathrm{L}$ in septic shock were associated with significant higher risk of 90-days mortality. In conclusion, PCT and MRproADM combination represents an advantage for sepsis diagnosis and for 90-days mortality risk stratification.
\end{abstract}

\section{Introduction}

Sepsis is a systemic syndrome induced by infection. The simultaneous presence of inflammation and infection contributes to organ failure pathogenesis with consequent complication of the clinical condition evolving towards septic shock, multi organ failure, and death [1,
2].

Sepsis and septic shock are characterized by high mortality rate ranging from $25-30 \%$ to $40-50 \%$ of patients, respectively [3]. Fast and correct diagnosis has a central role for the clinical management of sepsis, since delayed and inappropriate antibiotic therapy has a signoficant impact on patient prognosis [4,5]. Blood cultures represent the

\footnotetext{
Abbreviations: PCT, (procalcitonin); MR-proADM, (Mid-regional pro Adrenomedullin); TRACE, (time-resolved amplified cryptate emission); ROC, (Receiver operating characteristic); AUC, (Area under the curve); IQR, (Inter quartile range); BC, (blood cultures); BSI, (Blood stream infection); LPS, (lipopolysaccharides); TLR4, (toll-like receptor 4); LTA, (lipoteichoic acid); TLR2, (toll-like receptor 2); IL, (Interleukin); CLRs, (C-type lectin receptors); IFN, (Interferon); PPV, (Positive Predictive Value)

${ }^{*}$ Corresponding author.

E-mail address: s.angeletti@unicampus.it (S. Angeletti).
} 
gold standard for microbiological sepsis diagnosis [6], but can be time consuming and unable to assist physicians in the early therapeutic choices despite new diagnostic molecular techniques based on polymerase chain reaction and mass spectroscopy have been introduced to reduce time to pathogen detection $[7,8]$.

Besides these molecular approaches, that requiring specific equipment can be still expensive thus limiting widespread availability [9], recent biomarkers readily available as Procalcitonin (PCT) and Midregional pro Adrenomedullin (MR-proADM) have been proposed for sepsis diagnosis, prognosis and antibiotic therapy guidance [10-16].

PCT was described as useful marker of sepsis because it was able to discriminate between non-infectious and infectious inflammation with high sensitivity and specificity [11-15]. Moreover, some recent studies evaluated PCT ability to predict the etiological diagnosis associating specific cut-off values to gram-negative, gram-positive or fungi infection with the aim to guide early and appropriate antimicrobial empiric therapy [17-22]. Regarding MR-proADM, in some recent studies it was combined to PCT as marker of organ dysfunction [11-15,23-29].

In this study, PCT and MR-proADM were retrospectively analyzed as tools for etiological diagnosis of sepsis and septic shock and prognosis evaluation.

\section{Material and methods}

\subsection{Patients and settings}

The retrospective study was performed on 571 consecutively enrolled patients with diagnosis of sepsis or septic shock admitted at the University Hospital Campus Bio-Medico of Rome, Italy, between the years 2012-2018. The study was approved by the Local Ethical Committee and was performed according to the Declaration of Helsinki. Inclusion criteria were patients aged $\geq 18$ years with diagnosis of sepsis and/or septic shock microbiological documented by isolation of the causative pathogen from positive blood cultures. Exclusion criteria were patients with localized infection without clinical signs of sepsis or septic shock or with Systemic Infalmmatory Response Syndrome (SIRS) for not infectious causes. As control group, 340 plasma samples from 290 patients with not-infectious SIRS and 50 healthy individuals were collected for PCT and MR-proADM measurement.

Patient's demographic and clinical data were extracted from electronic clinical records.

\subsection{PCT and MR-proADM plasma measurement}

PCT and MR-proADM plasma concentrations were measured by time-resolved amplified cryptate emission (TRACE) technology with commercially available immunoluminometric assays assay (Brahms AG; Hennigsdorf, Germany) [10-14]. Plasma samples for PCT and MRproADM measurement were collected at the moment of clinical suspicious/diagnosis of sepsis following the Second and Third Consensus Conferences guidelines $[1,2,30]$.

\subsection{Statistical analysis}

Discrete demographic variables were compared using $\chi^{2}$ test, $p$ value $<0.05$ were considered as significant. PCT and MR-proADM values in patients with sepsis and control population were compared using the non-parametric Mann-Whitney's test, p value $<0.05$ were considered as significant. Receiver operating characteristic (ROC) analysis was performed to define the diagnostic ability of PCT and MRproADM and to define the cutoff point to use in sepsis diagnosis. Areas under the curve (AUCs) were calculated and compared for all variables. Pretest odds, posttest odds, and the consequent posttest probability have been calculated to evaluate advantage from biomarkers combination [31]. Data have been analyzed using Med-Calc 11.6.1.0 statistical package (MedCalc Software, Mariakerke, Belgium).
Table 1

Characteristic of the study population stratified for sepsis, septic shock and microbial etiology and of the control group.

\begin{tabular}{|c|c|}
\hline TOTAL SEPSIS & $571(100)$ \\
\hline SEPSIS & $342(60)$ \\
\hline SEPIC SHOCK & $229(40)$ \\
\hline Median age (25th-75th percentile) & $78\left(\mathrm{IQR}^{\mathrm{a}} 69-84\right)$ \\
\hline Male sex & $327(57)$ \\
\hline Female sex & $244(43)$ \\
\hline GRAM-POSITIVE SEPSIS & $171(30)$ \\
\hline S. agalactiae & $4(2)$ \\
\hline S. anginosus & $4(2)$ \\
\hline S. sanguinis & $3(1.7)$ \\
\hline S. gordonii & $2(1)$ \\
\hline S. pneumoniae & $2(1)$ \\
\hline E. faecalis & $26(15)$ \\
\hline E. faecium & $11(6.4)$ \\
\hline E. durans & $2(1)$ \\
\hline Staphylococcus aureus & $66(38.5)$ \\
\hline S. epidermidis & $17(10)$ \\
\hline S. hominis & $10(6)$ \\
\hline S. haemolyticus & $8(4.6)$ \\
\hline S. capitis & $4(2)$ \\
\hline S. hycus & $2(1)$ \\
\hline S. saprophyticus & $1(0.6)$ \\
\hline S. lugdunensis & $1(0.6)$ \\
\hline S. warneri & $1(0.6)$ \\
\hline GRAM-NEGATIVE SEPSIS & $260(45)$ \\
\hline E.coli & $127(49)$ \\
\hline K. pneumoniae & 49 (19) \\
\hline K. oxytoca & $7(2.6)$ \\
\hline E. cloacae & $12(5)$ \\
\hline E. aerogenes & $4(1.5)$ \\
\hline C. freundii & $4(1.5)$ \\
\hline S. marcescens & $5(1.9)$ \\
\hline P. mirabilis & $7(2.6)$ \\
\hline M. morganii & $2(0.7)$ \\
\hline A. baumannii & $3(1.0)$ \\
\hline$P$. aeruginosa & $31(12)$ \\
\hline S. maltophilia & $4(1.5)$ \\
\hline S. paucimobilis & $2(0.7)$ \\
\hline M. catarrhalis & $1(0.6)$ \\
\hline P. agglomerans & $1(0.6)$ \\
\hline R. planticola & $1(0.6)$ \\
\hline FUNGAL SEPSI & $50(9)$ \\
\hline Candida albicans & $31(62)$ \\
\hline Candida parapsilosis & $8(16)$ \\
\hline Candida tropicalis & $5(10)$ \\
\hline Candida glabrata & $4(8)$ \\
\hline Candida shaerica & $1(2)$ \\
\hline Candida norvegensis & $1(2)$ \\
\hline ANAEROBE (Bacteroides spp.) & $10(2)$ \\
\hline POLYMICROBIAL SEPSIS & $89(14)$ \\
\hline CONTROL GROUP & $340(100)$ \\
\hline Not-infectious SIRS & $290(85)$ \\
\hline Healthy individuals & $50(15)$ \\
\hline Median age & $74\left(\mathrm{IQR}^{\mathrm{a}} 67-81\right)$ \\
\hline Male sex & $201(59)$ \\
\hline Female sex & $139(41)$ \\
\hline
\end{tabular}

${ }^{\mathrm{a}} \mathrm{IQR}=$ interquartile range.

\section{Results}

\subsection{Patients}

Five hundreds and seventy-one patients with diagnosis of sepsis or septic shock admitted at the University Hospital Campus Bio-medico of 
Rome, in the years 2015-2018 were consecutively included in the study. The demographic and clinical characteristics of the study population are reported in Table 1 . The median age of the study and control populations were 78 years (Inter quartile range (IQR) 69-84) and 74 years (IQR 67-81), respectively. In the study group $327 / 571$ (57\%) were male and 229/571 (40\%) have diagnosis of septic shock (Table 1).

For all patients included in the study group, microorganisms were isolated from positive blood cultures (BCs) that in 482 (84\%) of cases were monomicrobial. BCs were positive for Gram-negative bacteria in $260 / 571$ (45\%), for Gram-positive in 171/571 (30\%), for fungal pathogens in 50/571 (95) and for anaerobe in 10/571(2\%) (Table 1). The most frequent microorganisms isolated were Escherichia coli $(127 / 571$, $22 \%$ ) and Staphylococcus aureus (66 isolates, 11.5\%) (Table 1).

In 89/571 (14\%) of patients, sepsis had a polymicrobial etiology, of these $42 / 89$ (47\%) presented sepsis and 47/89 (53\%) developed septic shock (Table 1). The polymicrobial sepsis was characterized in $35 / 42$ (83\%) of sepsis and $43 / 47$ (91\%) of septic shock by the presence of at least one Gram-negative pathogen (Table 1).

\subsection{PCT median values in patients with sepsis and septic shock and controls}

PCT median values have been reported in Table 2. In case of positive BCs, median values in sepsis and septic shock $(1.31 \mathrm{ng} / \mathrm{mL}$, IQR $0.66-3.90$ and $3.57 \mathrm{ng} / \mathrm{m}$ IQR $1.15-17.80$, respectively) were significantly higher $(\mathrm{p}<0.0001)$ than control population $(0.11 \mathrm{ng} / \mathrm{mL}$, IQR 0.06-0.23).

Stratifying patients by sepsis and septic shock, PCT median values were significantly higher $(\mathrm{p}<0.0001)$ in case of septic shock (3.57 ng/mL, IQR 1.15-17.8) (Table 2). In case of patient stratification by microbiological etiology, the highest PCT values were observed in Gram-negative BCs, especially in case of septic shock $(9.42 \mathrm{ng} / \mathrm{mL}$ 1.92-22.75). The lowest values were registered in Gram-positive and fungal sepsis $(0.64 \mathrm{ng} / \mathrm{mL}$, IQR 0.45-1.57; $0.86 \mathrm{ng} / \mathrm{mL}$, IQR 0.46-1.80, respectively), although values increased in case of septic shock the $(1.84 \mathrm{ng} / \mathrm{mL}$ and $1.16 \mathrm{ng} / \mathrm{mL}$, respectively). In polymicrobial septic shock, PCT showed the second highest median value $(5.22 \mathrm{ng} / \mathrm{mL}$, IQR 1.45-28.5) behind Gram-negative septic shock (Table 2).

By Mann-Whitney comparison, PCT median values in gram-negative BCs resulted significantly higher $(\mathrm{p}<0.0001)$ than gram-positive and fungal etiologies, whereas any significant difference was observed in case of anaerobe or polymicrobial sepsis (Fig. 1 panel A). Similarly, any significant difference was observed comparing gram-positive and fungal sepsis or septic shock (Fig. 1 panel A, Table 2).

Comparing PCT median values between septic and septic shock patients within the same microbial class, in any case PCT resulted significantly higher in septic shock, as reported in Table 2.

\subsection{MR-proADM median values in patients with sepsis and septic shock and controls}

Median values of MR-proADM have been reported in Table 2. In positive BCs, median values in sepsis or septic shock $(1.88 \mathrm{ng} / \mathrm{mL}$, IQR $1.33-2.84$ and $3.65 \mathrm{ng} / \mathrm{mL}$ IQR $2.35-6.91$, respectively) were significantly higher $(\mathrm{p}<0.0001)$ than control population $(0.74 \mathrm{ng} / \mathrm{mL}$, IQR 0.58-0.96) (Table 2 and Fig. 1 Panel B).

In septic patients, MR-proADM median values were mostly homogeneous and any significant difference was observed between the different microbial etiologies (Table 2, Fig. 1 panel B). Within the same microbial class, Mann-Whitney comparison showed median values significantly higher in septic shock than sepsis as reported in Table 2.

\subsection{PCT and MR-proADM in gram-negative blood stream infection (BSI)}

PCT median value was $3.74 \mathrm{ng} / \mathrm{mL}$ (IQR $2.53-5.89 \mathrm{ng} / \mathrm{mL}$ ). Stratifying by sepsis and septic shock, median values were significantly higher $(\mathrm{p}<0.0001)$ in case of septic shock $9.42 \mathrm{ng} / \mathrm{mL}$ (IQR $1.92-22.75 \mathrm{ng} / \mathrm{mL}$ ) than sepsis $2.33 \mathrm{ng} / \mathrm{mL}$ (IQR $0.96-10.80 \mathrm{ng} / \mathrm{mL}$ ) (Table 2). PCT values were evaluated also based on the different gramnegative species isolated in the study population as reported in Table 3. PCT was not significantly different comparing sepsis and septic shock within the same microbial class, except for Escherichia coli ( $\mathrm{p}=0.0001$ ) and Stenotrophomonas maltophilia $(\mathrm{p}=0.049)$ with higher values in septic shock (Table 3). PCT median values were significantly higher $(\mathrm{p}=0.0018)$ in presence of Enterobacteriaceae $(5.25 \mathrm{ng} / \mathrm{mL}, \quad$ IQR 1.32-19.2) than other gram-negative species $(1.77 \mathrm{ng} / \mathrm{mL}$, IQR 0.87-4.47) (Fig. 2 panel A). The highest values were registered in case of Proteus mirabilis isolates (34.40, IQR 6.40-35.00) and these were significantly higher than those found in case of Escherichia coli $(\mathrm{p}=0.032)$, Serratia marcescens $(\mathrm{p}=0.047)$ and Enterobacter cloacae ( $\mathrm{p}=0.028$ ) BSI (Fig. 3 panel B). Citrobacter freundii, Klebsiella oxytoca and Klebsiella pneumoniae showed rather high values but not significantly different from other Enterobacteriaceae species (Fig. 2 panel B).

Table 2

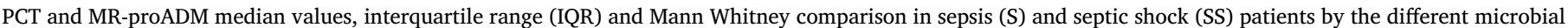
etiology and in negative blood cultures (BCs).

\begin{tabular}{|c|c|c|c|c|}
\hline & $\begin{array}{l}\text { PCT (ng/mL) } \\
\text { (IQR) }\end{array}$ & $\mathrm{p}^{\mathrm{a}}$ & $\begin{array}{l}\text { MR-proADM (nmol/L) } \\
\text { (IQR) }\end{array}$ & $\mathrm{p}^{\mathrm{a}}$ \\
\hline Sepsis & $1.31(0.66-3.90)$ & $<0.0001$ & $1.88(1.33-2.84)$ & $<0.0001$ \\
\hline Septic Shock & $3.57(1.15-17.80)$ & & $3.65(2.35-6.91)$ & \\
\hline Gram-negative BSI & $3.74(2.53-5.89)$ & & $2.46(1.50-4.00)$ & \\
\hline Gram-negative sepsis & $2.33(0.96-10.80)$ & $<0.0001$ & $1.73(1.32-2.66)$ & $<0.0001$ \\
\hline Gram-negative SS & $9.42(1.92-22.75)$ & & $3.79(2.45-7.31)$ & \\
\hline Gram-positive BSI & $0.76(0.48-2.02)$ & & $2.04(1.39-3.38)$ & \\
\hline Gram-positive sepsis & $0.64(0.45-1.57)$ & 0.03 & $2.09(1.27-2.57)$ & $<0.0001$ \\
\hline Gram-positive SS & $1.16(0.53-3-34)$ & & $3.44(2.10-7.48)$ & \\
\hline Fungal BSI & $1.20(0.55-2.85)$ & & $2.96(1.90-4.97)$ & \\
\hline Fungal sepsis & $0.86(0.46-1.80)$ & 0.01 & $2.31(1.55-3.16)$ & 0.0012 \\
\hline Fungal SS & $1.96(0.79-6.71)$ & & $4.51(2.43-7.71)$ & \\
\hline Polymicrobial BSI & $2.53(0.97-12.00)$ & & $2.60(1.59-4.93)$ & \\
\hline Polymicrobial sepsis & $1.98(0.92-5.66)$ & 0.01 & $2.06(1.30-3.50)$ & 0.0033 \\
\hline Polymicrobial SS & $5.22(1.45-28.5)$ & & $3.50(1.72-5.80)$ & \\
\hline Anaerobe BSI & $2.18(0.55-3.08)$ & & $1.92(1.6-5.08)$ & \\
\hline Anaerobe sepsis & $2.37(1.12-3.08)$ & ns & $2.04(1.72-4.47)$ & ns \\
\hline Anaerobe SS & $3.90(0.49-27.0)$ & & $1.60(1.48-9.49)$ & \\
\hline Control group & $0.11(0.06-0.21)$ & & $0.74(0.58-0.96)$ & \\
\hline
\end{tabular}

a Mann Whitney comparison of median values. 
$\mathbf{A}$

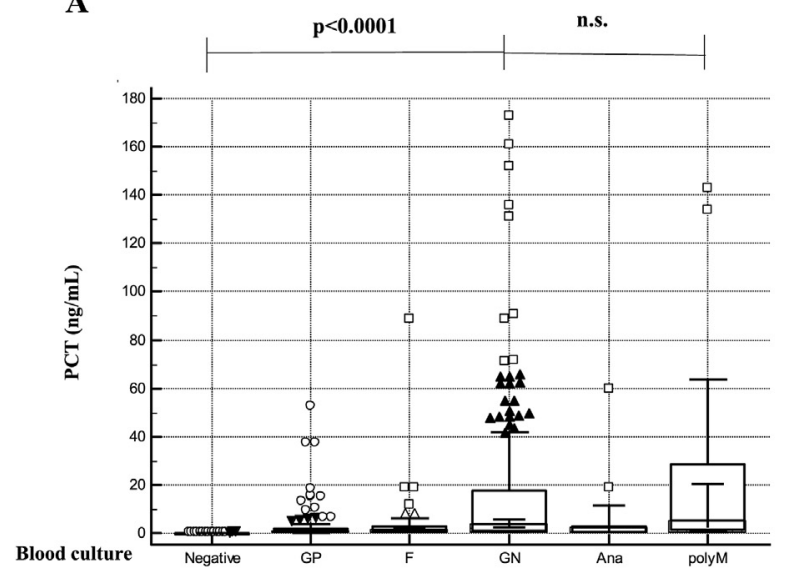

B

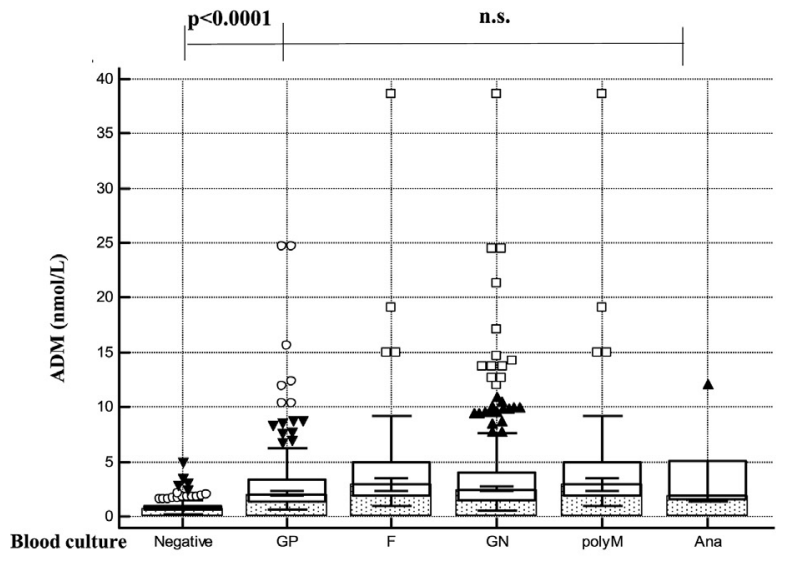

Fig. 1. PCT median values according to BC result: Mann-Whitney comparison between gram-negative (GN) vs, gram-positive (GP), fungi (F), anaerobes (Ana), polymicrobial (polyM) and negative BCs (panel A); ADM median values according to BC result: Mann-Whitney comparison between Fungi (F) vs negative, grampositive (GP), gram-negative (GN), anaerobes (Ana) and polymicrobial (polyM) BCs (panel B).

In Gram-negative BSI, MR-proADM median value was $2.46 \mathrm{nmol} / \mathrm{L}$ (IQR $1.50-4.00 \mathrm{nmol} / \mathrm{L}$ ). Stratifying by sepsis and septic shock, median values were significantly higher $(\mathrm{p}<0.0001)$ in case of septic shock $3.65 \mathrm{nmol} / \mathrm{L}$ (IQR $2.35-6.91 \mathrm{nmol} / \mathrm{L}$ ) than sepsis $1.88 \mathrm{nmol} / \mathrm{L}$ (IQR (1.33-2.84 nmol/L) (Table 2). MR-proADM median values were analyzed for different gram-negative species isolated in the study population as reported in Table 3. Significant differences between sepsis and septic shock in case of Escherichia coli ( $\mathrm{p}<0.0001$ ), Pseudomonas aeruginosa $(\mathrm{p}=0.0001)$ and Klebsiella pneumoniae $(\mathrm{p}=0.0009)$ were found with highest values in septic shock (Table 3). Any significant difference between Enterobacteriaceae species $(2.40 \mathrm{nmol} / \mathrm{L}, \mathrm{IQR}$ 1.48-3.94) and other gram-negative species $(2.93 \mathrm{nmol} / \mathrm{L}$, IQR $1.52-4.80 \mathrm{nmol} / \mathrm{L}$ ) was observed (Fig. 2 panel C). Within Enterobacteriaceae, the highest MR-proADM median values were found in case of Citrobacter freundii BSI $(5.21 \mathrm{nmol} / \mathrm{L}$, IQR $4.96-7.48 \mathrm{nmol} / \mathrm{L})$, whereas in presence of Proteus mirabilis the lowest median values (1.80 nmol/L, IQR $1.48-2.32 \mathrm{nmol} / \mathrm{L})$ were observed. A statistical significant difference was observed between MR-proADM values in case of Citrobacter freundii isolates and Proteus mirabilis $(\mathrm{p}=0.0082)$, Escherichia coli $(\mathrm{p}=0.027)$, Serratia marcescens $(\mathrm{p}=0.0082)$, Enterobacter cloacae $(\mathrm{p}=0.0082)$ and Klebsiella oxytoca $(\mathrm{p}=0.0082)$, whereas any significant difference was observed between Citrobacter freundii and Klebsiella pneumoniae (Fig. 2 panel D).

\subsection{PCT and MR-proADM median values in gram-positive BSI}

PCT median value was $2.04 \mathrm{nmol} / \mathrm{L}$ (IQR $(1.39-3.38 \mathrm{ng} / \mathrm{mL}$ ). Stratifying by sepsis and septic shock, median values were significantly higher $(\mathrm{p}<0.0001)$ in case of septic shock $3.44 \mathrm{nmol} / \mathrm{L}$ (IQR $2.10-7.48 \mathrm{nmol} / \mathrm{L}$ ) than sepsis $2.09 \mathrm{nmol} / \mathrm{L}$ (IQR $1.27-2.57 \mathrm{nmol} / \mathrm{L}$ ) (Table 2).

PCT median values detected in different gram-positive species isolated from blood in septic patients are reported in Table 3. The highest values were registered in case of S.aureus sepsis (Table 3 and Fig. 3 panel A and B). Statistically significant highest values are detected comparing $S$. aureus and Enterococcus spp versus Coagulase negative Staphylococcus (ConS) $(\mathrm{p}=0.0003$ and $\mathrm{p}=0.0120$ respectively). Any significant difference was evidenced between $S$. aureus, Enteroccoccus and Streptococcus spp and between Streptococcus spp. and ConS. In case of Streptococcus spp., PCT median values were not statistically different from all other gram-positive isolates (Fig. 3 panel A and B). Stratifying patients by sepsis and septic shock, none significant difference was evidenced, except for $S$. aureus isolates showing highest values in case of septic shock $(\mathrm{p}=0.03)$ (Table 3$)$ and confirming the significant difference with ConS septic shock $(\mathrm{p}=0.0115)$.
In Gram-positive BSI, MR-proADM median value was $2.04 \mathrm{nmol} / \mathrm{L}$ (IQR $1.39-3.38 \mathrm{nmol} / \mathrm{L}$ ). Stratifying by sepsis and septic shock, median values were significantly higher $(\mathrm{p}<0.0001)$ in case of septic shock $3.44 \mathrm{nmol} / \mathrm{L}$ (IQR $2.10-7.48 \mathrm{nmol} / \mathrm{L}$ ) than sepsis $2.09 \mathrm{nmol} / \mathrm{L}$ (IQR $(1.27-2.57 \mathrm{nmol} / \mathrm{L})$ (Table 2). MR-proADM median values were analyzed for different gram-positive isolates of the study population as reported in Table 3. Significant differences between sepsis and septic shock in case of $S$. aureus $(\mathrm{p}=0.0012)$, Enterococcus spp. $(\mathrm{p}=0.0037)$ and Streptococcus spp. $(\mathrm{p}=0.050)$ were found with highest values in septic shock, whereas any significant difference was observed in case of Cons (Table 3).

Even if MR-proADM values were homogeneously distributed between the different species, the highest values were observed in case of Enterococcus spp (5.34 nmol/L (IQR 2.03-7.82 nmol/L) (Fig. 3 panel C and D).

\subsection{PCT and MR-proADM median values in fungal BSI}

In fungal BSIs, PCT median value was $1.20 \mathrm{nmol} / \mathrm{L}$ (IQR $(0.55-2.85 \mathrm{ng} / \mathrm{mL})$. Stratifying by sepsis and septic shock, median values were significantly higher $(\mathrm{p}=0.01)$ in case of septic shock $(1.96 \mathrm{nmol} / \mathrm{L} ; \mathrm{IQR} 0.79-6.71 \mathrm{nmol} / \mathrm{L})$ than sepsis $(0.86 \mathrm{nmol} / \mathrm{L} \mathrm{IQR}$ $0.46-1.80 \mathrm{nmol} / \mathrm{L}$ ) (Table 2). This was observed especially in case of Candida albicans isolates (Table 3).

PCT median values for the different blood fungal isolates are reported in Table 3. The highest values were registered in case of Candida tropicalis isolates $(2.85 \mathrm{nmol} / \mathrm{L}$, IQR $0.55-6.74 \mathrm{nmol} / \mathrm{L}$ ) (Table 3 and Fig. 4 panel A and B), whereas any significant difference was observed between the different fungal isolates. MR-proADM values were homogeneously distributed between the different fungal species, whereas the highest values were observed in case of Candida albicans $(3.12 \mathrm{nmol} / \mathrm{L}$ (IQR $2.50-6.95 \mathrm{nmol} / \mathrm{L}$ ). However, any significant difference was observed between the species isolates (Fig. 4 panel C and D). Low PCT values were registered especially in case of Candida parapsilosis $(0.58 \mathrm{nmol} / 1 \mathrm{IQR} 0.42-0.88 \mathrm{nmol} / \mathrm{L})$ and Candida glabrata $(0.59 \mathrm{nmol} / \mathrm{L}$ IQR $0.33-1.22$ nomol/L) isolates and this trend was observed also for MR-proADM values that in presence of these isolates resulted the lowest $(1.70 \mathrm{nmol} / \mathrm{L}, \quad$ IQR $\quad 1.43-3.72 \mathrm{nmol} / \mathrm{L}$ and $2.00 \mathrm{nmol} / \mathrm{L}, \quad$ IQR $1.56-2.58 \mathrm{nmol} / \mathrm{L}$, respectively) (Table 3 ).

\subsection{PCT and MR-proADM median values in polymicrobial BSI}

In case of polymicrobial etiology, highest PCT median values were detected when the polymicrobic component consisted exclusively of different gram-negative isolates $(6.28 \mathrm{ng} / \mathrm{mL}$, IQR $2.12-36.00 \mathrm{ng} / \mathrm{mL})$, 
Table 3

Median values, interquartile range (IQR) and Mann Whitney comparison of PCT and MR-proADM in monomicrobial and polymicrobial sepsis and septic shock by the different microorganism isolated from blood.

MONOMICROBIAL sepsis and septic shock

\begin{tabular}{|c|c|c|c|c|c|}
\hline Gram-negative isolates & PCT (IQR) (ng/mL) & & MR-proADM (IQR) (nmol/L) & & \\
\hline E. coli & $3.4(1.13-14.0)$ & & $2.40(1.46-3.77)$ & & \\
\hline E. coli $S$ & $1.86(0.99-9.17)$ & $\mathrm{p}=0.0001$ & $1.73(1.32-2.66)$ & & $\mathrm{p}<0.0001$ \\
\hline E. coli SS & $12.90(2.66-28.85)$ & & $4.20(2.43-9.56)$ & & \\
\hline K. pneumoniae & $8.2(1.90-24.54)$ & & $2.92(1.74-5.67)$ & & \\
\hline$K$. pneumoniae $S$ & $3.70(0.95-28.32)$ & n.s. & $1.80(1.50-3.07)$ & & $\mathrm{p}=0.0004$ \\
\hline K. pneumoniae SS & $11.0(2.84-22.90)$ & & $4.51(2.62-6.28)$ & & \\
\hline K. oxytoca & $17.4(13.04-22.00)$ & & $2.25(1.10-3.05)$ & & \\
\hline K. oxytoca $S$ & $17.41(13.04-25.55)$ & n.s. & $2.25(0.81-2.51)$ & & n.s. \\
\hline K. oxytoca SS & $14.20(6.53-22.00)$ & & $2.54(1.77-3.32)$ & & \\
\hline P. aeruginosa & $1.70(0.87-3.31)$ & & $3.42(1.60-5.59)$ & & \\
\hline$P$. aeruginosa $\mathrm{S}$ & $1.17(0.86-4.03)$ & n.s. & $1.69(1.14-3.35)$ & & $\mathrm{p}=0.0009$ \\
\hline P. aeruginosa SS & $1.92(1.03-4.15)$ & & $4.92(3.45-8.28)$ & & \\
\hline P. mirabilis & $34.40(6.40-35.00)$ & & $1.80(1.48-2.32)$ & & \\
\hline P. mirabilis $S$ & $34.4(6.4-35.0)$ & n.s. & $1.80(1.48-1.97)$ & & n.s. \\
\hline P. mirabilis SS & $46.8(2.90-90.8)$ & & $2.47(1.36-3.60)$ & & \\
\hline E. cloacae & $4.15(1.13-22.76)$ & & $2.11(1.27-3-05)$ & & \\
\hline E. cloacae $S$ & $1.44(1.07-15.72)$ & n.s. & $1.38(1.09-2.54)$ & & n.s. \\
\hline E. cloacae SS & $6.92(3.16-43.09)$ & & $2.82(1.76-12.13)$ & & \\
\hline C. freundii & $19.2(15.57-21.30)$ & & $5.21(4.96-7.48)$ & & \\
\hline C. freundii $S$ & $20.67(19.17-22.67)$ & n.s. & $4.94(4.92-4.96)$ & & n.s. \\
\hline C. freundii SS & $17.71(11.98-23.44)$ & & $7.48(5.97-9.50)$ & & \\
\hline S. marcescens & $4.28(2.16-12.57)$ & & $2.55(2.37-2.85)$ & & \\
\hline S. marcescens $S$ & $4.28(4.04-4.59)$ & n.s. & $2.50(2.23-2.76)$ & & n.s. \\
\hline S. marcescens SS & $8.38(1.53-17.70)$ & & $2.70(2.44-3.23)$ & & \\
\hline S. maltophilia & $2.18(0.56-3.50)$ & & $1.54(0.66-2.35)$ & & \\
\hline S. maltophilia $S$ & $0.56(0.48-0.86)$ & 0.049 & $0.66(0.66-0.86)$ & & 0.049 \\
\hline S. maltophilia SS & $3.50(3.42-3.72)$ & & $2.35(2.20-2.50)$ & & \\
\hline S. paucimobilis SS & $35.2(20.6-49.8)$ & & $2.38(2.35-2.41)$ & & \\
\hline Gram-positive isolates & PCT (IQR) (ng/mL) & & MR-proADM (IQR) (nmol/L) & & \\
\hline S. aureus & $1.31(0.54-2.70)$ & & $2.06(1.50-3.44)$ & & \\
\hline S. aureus $S$ & $1.15(0.54-1.84)$ & $\mathrm{p}=0.03$ & $1.82(1.27-2.23)$ & $\mathrm{p}=0.0012$ & \\
\hline S. aureus SS & $2.70(0.55-8.85)$ & & $3.47(2.44-8.80)$ & & \\
\hline Enterococcus spp. & $0.80(0.51-2.26)$ & & $2.19(1.54-5.01)$ & & \\
\hline Enterococcus spp. $S$ & $0.68(0.51-2.40)$ & n.s. & $1.30(130-2.60)$ & $\mathrm{P}=0.0037$ & \\
\hline Enterococcus spp. SS & $1.05(0.46-2.16)$ & & $5.34(2.03-7.82)$ & & \\
\hline Streptococcus spp. & $0.80(0.39-2.06)$ & & $1.49(1.23-2.67)$ & & \\
\hline Streptococcus spp. $S$ & $0.50(0.38-1.94)$ & n.s & $1.38(1.18-2.05)$ & $\mathrm{p}=0.05$ & \\
\hline Streptococcus spp. SS & $1.40(0.83-6.91)$ & & $3.25(2.24-3.73)$ & & \\
\hline Coagulase negative Staphylococcus (ConS) & $0.52(0.32-0.73)$ & & $2.21(1.39-2.94)$ & & \\
\hline Cons $S$ & $0.47(0.31-0.86)$ & n.s & $2.12(1.39-2.81)$ & n.s & \\
\hline Cons SS & $0.61(0.32-0.67)$ & & $2.91(1.55-3.95)$ & & \\
\hline S. epidermidis $S$ & $0.37(0.30-0.56)$ & n.s. & $1.81(1.39-2.15)$ & n.s. & \\
\hline S. epidermidis SS & $0.63(0.39-0.68)$ & & $2.12(1.13-3.34)$ & & \\
\hline S. haemolyticus $S$ & $1.46(0.36-2.05)$ & n.s. & $2.72(2.30-3.40)$ & n.s. & \\
\hline S. haemolyticus SS & $0.65(0.41-1.06)$ & & $4.15(3.07-6.28)$ & & \\
\hline Others $^{\mathrm{a}}$ & $0.55(0.45-0.67)$ & & $1.23(1.20-2.37)$ & & \\
\hline Fungal isolates & PCT (IQR) (ng/mL) & & MR-proADM (IQR) (nmol/L) & & \\
\hline C. albicans & $1.80(0.94-3.53)$ & & $3.12(2.50-6.95)$ & & \\
\hline C. albicans $S$ & $1.03(0.55-1,08)$ & $\mathrm{p}=0.013$ & $2.53(1.90-3.12)$ & $\mathrm{p}=0.016$ & \\
\hline C. albicans SS & $2.84(1.30-9.56)$ & & $6.30(3.02-10.66)$ & & \\
\hline C. parapsilosis & $0.58(0.42-0.88)$ & & $1.70(1.43-3.72)$ & & \\
\hline C. parapsilosis $S$ & $0.47(0.38-0.80)$ & n.s. & $1.49(1.43-3.40)$ & n.s. & \\
\hline C. parapsilosis SS & $0.82(0.67-0.97)$ & & $2.95(1.86-4.05)$ & & \\
\hline C. tropicalis & $2.85(0.55-6.74)$ & & $2.40(1.81-2.69)$ & & \\
\hline C. tropicalis $S$ & $2.85(1.12-5.37)$ & n.s. & $2.40(2.02-3.28)$ & n.s. & \\
\hline C. tropicalis SS & $4.45(0.55-8.35)$ & & $1.98(1.57-2.40)$ & & \\
\hline C. glabrata & $0.59(0.33-1.22)$ & & $2.00(1.56-2.58)$ & & \\
\hline C. glabrata $S$ & $0.97(0.33-1.61)$ & n.s. & $2.14(1.13-3.16)$ & n.s. & \\
\hline C. glabrata SS & $0.59(0.34-0.84)$ & & $2.13(2.02-2.25)$ & & \\
\hline
\end{tabular}

POLYMICROBIAL sepsis and septic shock

Polymicrobial GN ${ }^{\mathrm{b}}$

Polymicrobial GN Sepsis Polymicrobial GN SS

Polymicrobial GN-GP

Polymicrobial GN-GP S

Polymicrobial GN-GP SS

Polymicrobial GP
PCT (IQR) (ng/mL)

$6.28(2.12-36.00)$

$3.93(1.53-5.50)$

$24.90(2.36-51.00)$

$4.67(0.97-11.35)$

$5.58(0.97-11.07)$

$4.40(1.31-23.60)$

$0.85(0.53-2.48)$
MR-proADM (IQR)

$3.44(1.36-5.72)$

$1.36(1.20-2.76)$

$5.21(1.70-5.80)$

$2.58(1.65-4.73)$

$2.46(1.65-3.74)$

$2.63(2.02-6.95)$

$1.99(1.58-5.00)$ p

$\mathrm{p}=0.003$

n.s. 
Table 3 (continued)

\begin{tabular}{|c|c|c|c|c|}
\hline Polymicrobial GP S & $0.56(0.41-2.03)$ & n.s. & $1.91(1.26-3.79)$ & n.s. \\
\hline Polymicrobial GP SS & $1.59(0.69-2.43)$ & & $3.56(2.04-5.00)$ & \\
\hline Polymicrobial Y-GN-GP ${ }^{\mathrm{b}}$ & $1.12(0.67-2.13)$ & & $2.02(1.50-3.66)$ & \\
\hline Polymicrobial Y-GN-GP S & $0.98(0.66-1.73)$ & n.s. & $1.92(1.25-3.14)$ & n.s. \\
\hline Polymicrobial Y-GN-GP SS & $1.27(0.71-12.72)$ & & $3.38(1.58-6.12)$ & \\
\hline
\end{tabular}

a S hominis; S.capitis S. hycus; S. saprophyticus; S. lugdunensis; S. warneri).

b Mann Whitney comparison: Polymicrobial Gram-negative vs Polymicrobial Gram-positive p = 0.0048; Polymicrobial Gram-negative vs Polymicrobial Fungi/

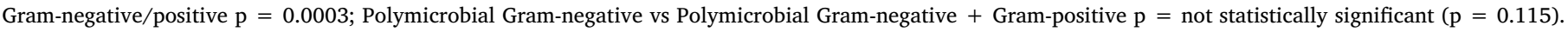

also stratifying by sepsis and septic shock with significantly higher values in septic shock ( $\mathrm{p}<0.0001$ ) (Table 3$)$. The lowest PCT values where registered when the polymicrobial component consisted exclusively of Gram-positive microorganisms $(0.85 \mathrm{ng} / \mathrm{mL}$, IQR $0.53-2.48 \mathrm{ng} / \mathrm{mL}$ ) (Table 3). This difference between polymicrobial pure gram-negative sepsis and polymicrobial pure gram-positive BSI resulted statistically significant $(\mathrm{p}=0.0048$ ). When the polymcrobial component was mixed and composed of gram-negative, gram-positive and yeast isolates, PCT values significantly increased due to the gramnegative component ( $\mathrm{p}=0.0137$ ) (Table 3$)$.

Regarding MR-proADM, even if the highest median values were registered in pure Gram-negative polymicrobial sepsis $(3.44 \mathrm{ng} / \mathrm{mL}$, IQR 1.36-5.72) and the lowest in pure Gram-positive (1.99 ng/mL, IQR $1.58-5.00 \mathrm{ng} / \mathrm{mL}$ ), any significant difference was found between the different polymicrobial isolates (Table 3 ).

\subsection{ROC curve analysis}

By ROC curve analysis the diagnostic ability of PCT and MRproADM has been tested independently from microbial etiology and the diagnostic cut-off for both markers have been calculated. Cut-off values resulted $>0.5 \mathrm{ng} / \mathrm{mL}$ for PCT and $>1 \mathrm{nmol} / 1$ for MR-proADM in sepsis, whereas in case of septic shock the cut-off of MR-proADM was

A

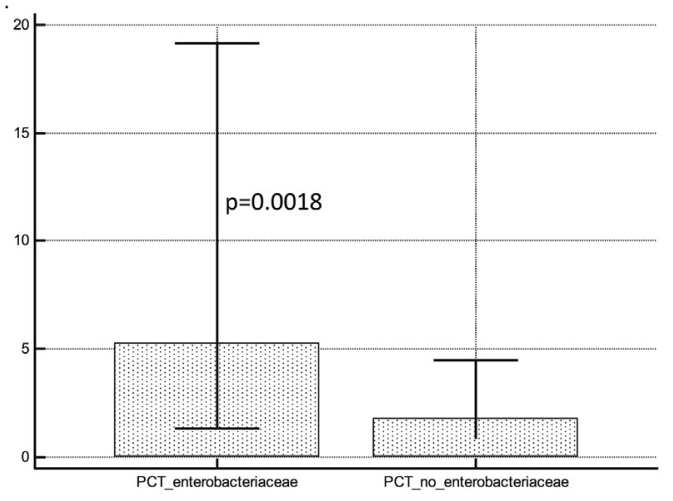

B

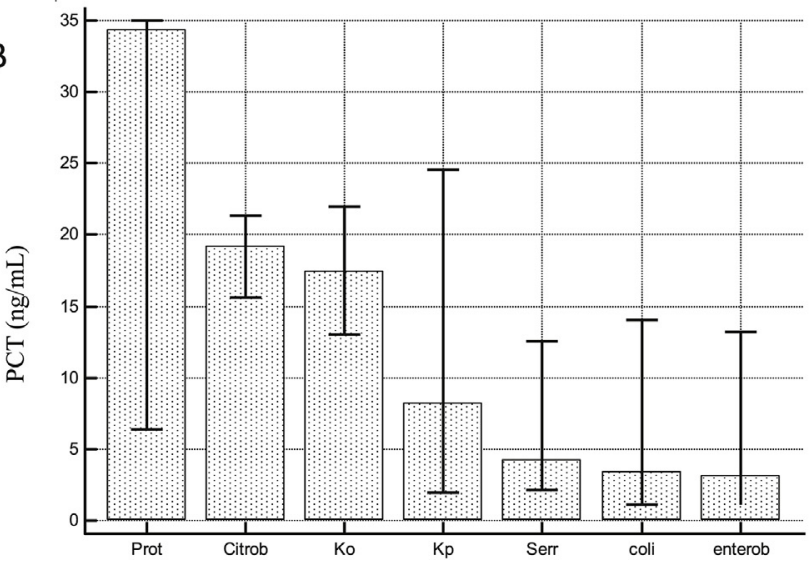

slightly increased $(>1.34 \mathrm{nmol} / \mathrm{L})$. Highest cut-off for both markers, $>2.7 \mathrm{ng} / \mathrm{ml}$ and $>2.7 \mathrm{nmol} / \mathrm{L}$ could be used to evidence evolution from sepsis towards septic shock (Table 4). PCT and MR-proADM AUC comparison in sepsis and septic shock showed a significant higher diagnostic ability of PCT to identify septic patients $(\mathrm{p}<0.0001)$ (Fig. 5 panel A). A significant higher AUC for MR-proADM was found to discriminate between sepsis and septic shock patients $(p<0.0001)$ (Fig. 5 panel B), whereas any significant difference between the PCT and MR-proADM AUCs was observed to identify septic shock patients (Fig. 5 panel C).

ROC curve analysis for PCT and MR-proADM was further extended in function of the different microbial etiology (Table 4). In case of Gram-negative bacteria, PCT cut-off of was $>0.5 \mathrm{ng} / \mathrm{mL}$ in sepsis and $>0.65 \mathrm{ng} / \mathrm{ml}$ in septic shock, PCT cut-off were $>0.3 \mathrm{ng} / \mathrm{mL}$ in gram-positive and fungal sepsis and $>0.45 \mathrm{ng} / \mathrm{ml}$ in fungal septic shock, (Table 4). In case of polymicrobial etiology, PCT cut off values were halfway between gram-positive/fungal and gram-negative etiologies $>0.42 \mathrm{ng} / \mathrm{mL}$ and $>0.53$ in sepsis and septic shock, respectively (Table 4).

In monomicrobial BSI, it was possible to identify a discriminating cut-off between the different species (Table 5). PCT cut-off values between gram-negative and gram-positive sepsis or septic shock were $>$ $0.80 \mathrm{ng} / \mathrm{mL}$ and $>3.88 \mathrm{ng} / \mathrm{mL}$ (Fig. 6 panel A and B), respectively.

Fig. 2. Mann-Whitney comparison of PCT (panel A and B) and MR-proADM (panel C and D) median values in Enterobacteriaceae spp. 

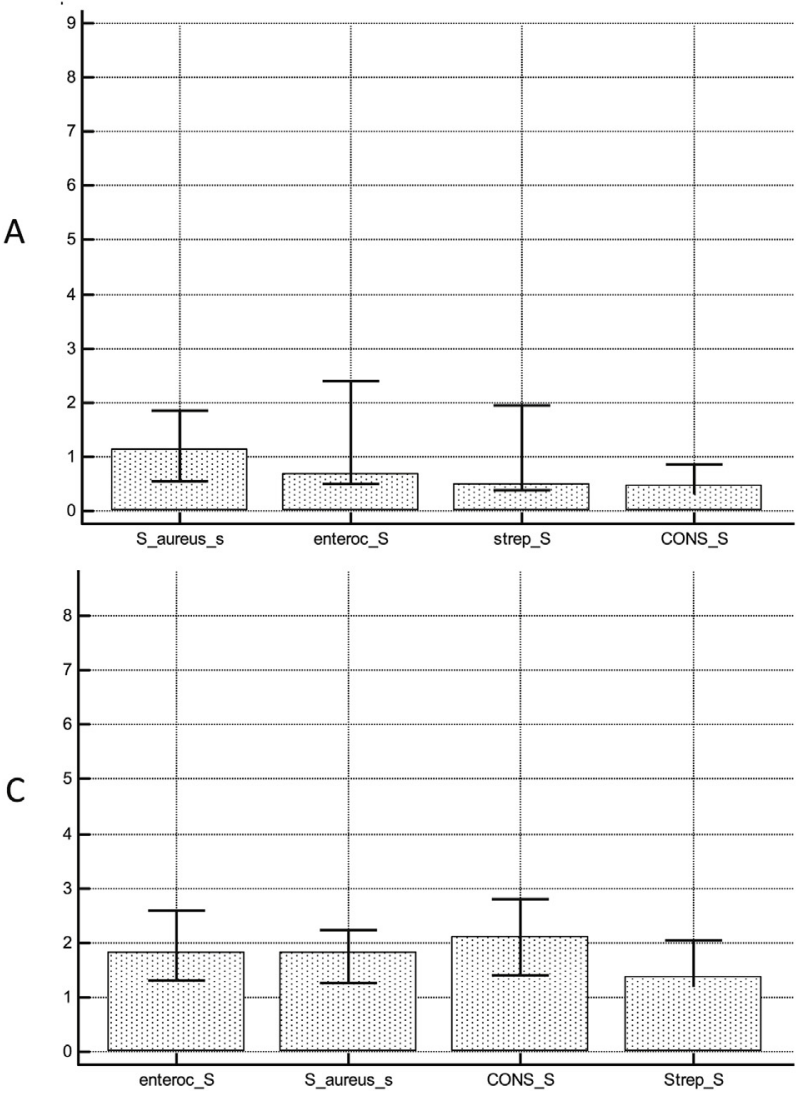
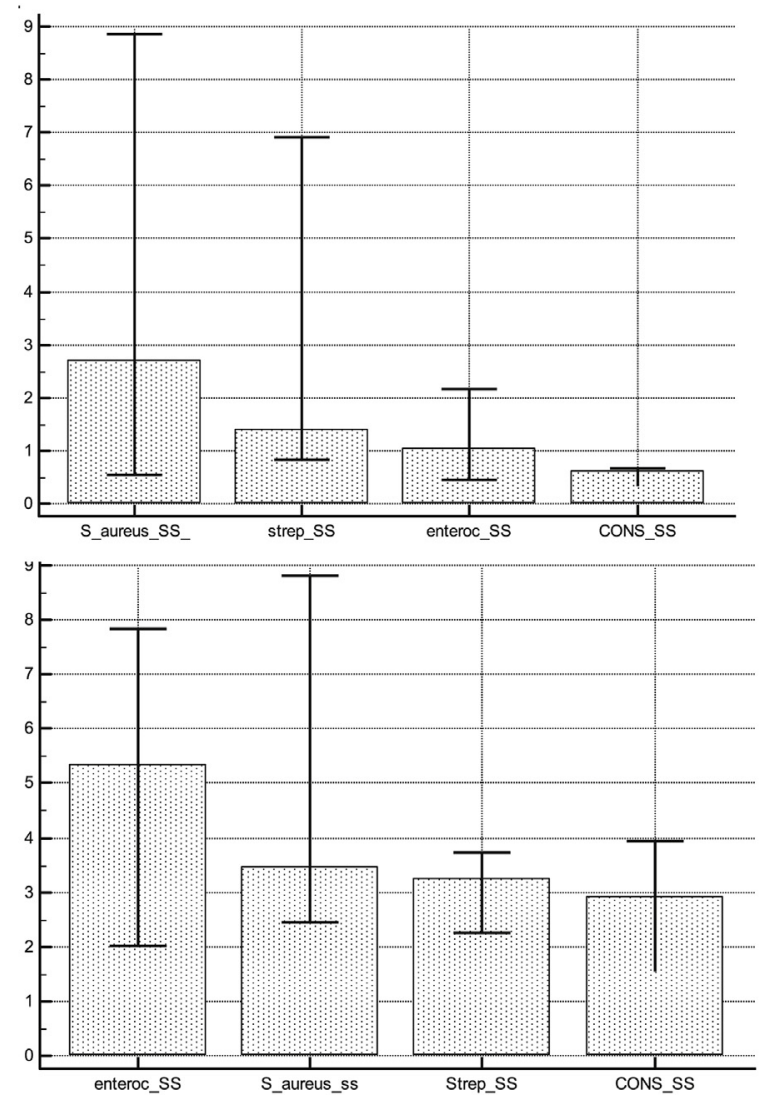

D

Fig. 3. Mann-Whitney comparison PCT (panel A e B) and MR-proADM of median values (panel C and D) in Gram-positive sepsis (S) and septic shock (SS).

Gram-negative and fungal sepsis or septic shock were discriminated with the highest Positive Predictive Value (PPV) if PCT was $>3.57 \mathrm{ng}$ / $\mathrm{mL}$ and $>8.77 \mathrm{ng} / \mathrm{mL}$, respectively (Fig. 6 panel C and D, Table 5). Regarding gram-positive discrimination from fungal sepsis, the cut-off values were $>0.56 \mathrm{ng} / \mathrm{mL}$ in sepsis with the lowest PPV and $>1.74$ $\mathrm{ng} / \mathrm{mL}$ in septic shock (Fig. 7 panel A and B, Table 5).

MR-proADM cut-off were homogeneously distributed in Gram-negative and Gram-positive etiology, being $>1 \mathrm{nmol} / \mathrm{L}$ in case of sepsis and within a range from 1.34 to $1.50 \mathrm{nmol} / \mathrm{L}$ for septic shock. Noteworthy, in fungal sepsis and septic shock the cut-off were increased ( $>1.37$ and $1.56 \mathrm{nmol} / \mathrm{L}$, respectively) (Table 5).

\subsection{MR-proADM and 90-days mortality prediction}

In a subset of $210 / 571$ septic patients (122 with sepsis and 88 with septic shock), ROC curve analysis was used to analyze MR-proADM and 90-days mortality. Cut-off values $>3.39 \mathrm{nmol} / \mathrm{L}$ in sepsis and $>4.33$ nmol/L in septic shock (Fig. 8 panel A and B) were associated with a significant higher risk of 90-days mortality, corresponding to odds ratio of 5.84 (95\% CI 2.90-11.74, p $<0.0001)$ and $6.17(95 \%$ CI 2.41-15.74, $\mathrm{p}<0.0001)$, respectively.

\section{Discussion}

In this study, the role of PCT and MR-proADM in the etiological diagnosis and prognosis of sepsis and septic shock has been evaluated.

Stratifying patients on microbial etiology, PCT resulted excellent in case of gram-negative sepsis and septic shock diagnosis but less performant in case of gram-positive and fungal etiologies. This lower release could be due to the different Toll-like receptor signaling pathways activation producing different pro-inflammatory cytokines [20].

In the immune response, Gram-negative bacteria lipopolysaccharides (LPS), is recognized by toll-like receptor 4 (TLR4), while Gram- positive lipoteichoic acid (LTA) by toll-like receptor 2 (TLR2) resulting in different secretion of inflammatory cytokines. Higher levels of Interleukin (IL)- 6 and IL-8 have been reported in case of Gram-negative sepsis than Gram-positive or fungal sepsis [32-35].

Fungal pathogens are recognized by C-type lectin receptors (CLRs) inducing the release of cytokines as IL-1 $\beta$, IL-10, and IL-6, resulting in moderately elevated levels of PCT that is influenced by Interferon (IFN)- $\gamma$ inhibition [36-40] and its relative levels.

All these factors probably contributed to the observed differences in PCT values reported in gram-negative, gram-positive and fungal sepsis $[20,41,42]$.

MR-proADM values resulted homogenously distributed within the different microbial classes. Median values tend to increase significantly when sepsis was complicated towards septic shock. These data further confirm the prognostic role of this marker in septic patients, as previously reported [11-15]. In case of gram-negative, PCT values were significantly different between sepsis and septic shock only in case of $E$. coli and $S$. maltophilia, whereas MR-proADM was significantly higher in septic shock also in K. pneumoniae and P. aeruginosa BSI. This could suggest that while PCT is a good diagnostic marker, it is not useful enough at prognostic purpose being influenced by the microbial etiology [41,42], in fact sepsis by Enterobacteriaceae are characterized by the highest PCT values. Within this group, in case of Proteus mirabilis PCT showed the highest values that is in line with other studies [17-23,43]. Conversely, MR-proADM was not different between Enterobacteriaceae and other gram-negative pathogens; its values were significantly higher in case of Citrobacter spp. and Klebsiella spp. suggesting that these pathogens could induce more severe sepsis than other gram-negative isolates, thus confirming the prognostic role of the biomarker [23-29,43]. These results indicate that in gram-negative sepsis PCT and MR-proADM could give complementary information to be used at diagnostic as well as at prognostic purpose.

In gram-positive BSI, the highest PCT values were detected in case 

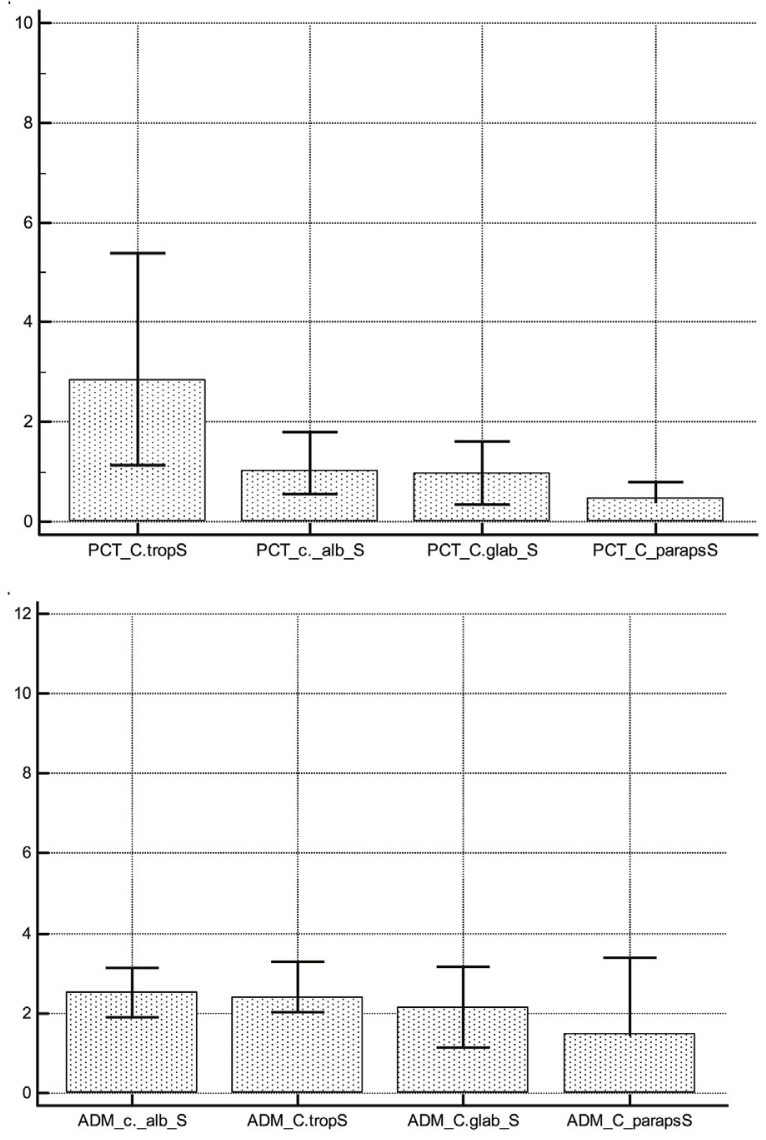
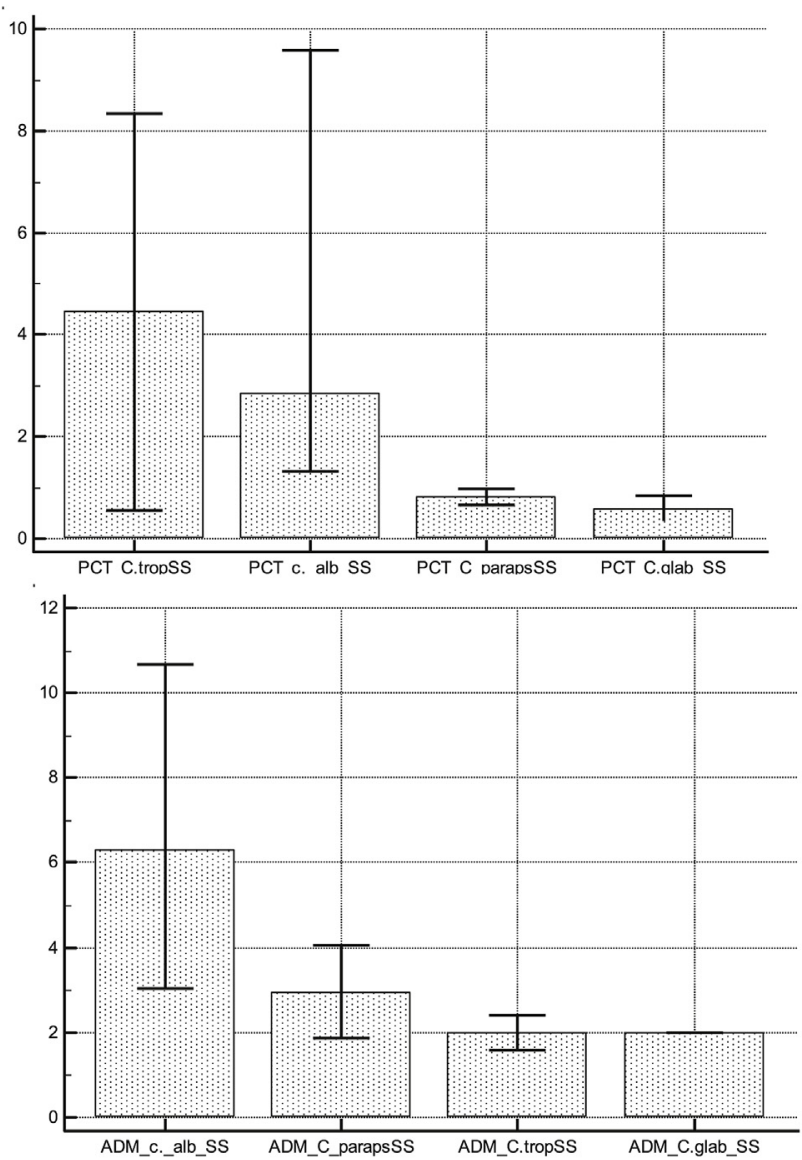

Fig. 4. Mann-Whitney comparison PCT (panel A and B) and MR-proADM of median values (panel C and D) in fungal sepsis (S) and septic shock (SS).

Table 4

Receiver operating characteristic (ROC) curves: areas under the curves (AUCs) and cutoff values for PCT and MR-proADM, in the diagnosis sepsis (S) and septic shock (SS) in monomicrobial gram-negative, gram-positive, fungal and polymicrobial bloodstream infection.

\begin{tabular}{|c|c|c|c|c|c|}
\hline \multicolumn{6}{|c|}{ Septic and Septic Shock patients versus control population } \\
\hline & PCT Cut-off & MR-proADM Cut-off & PCT AUCs & MR-proADM AUCs & $\mathrm{p} \Delta \mathrm{AUCs}$ \\
\hline All sepsis & $>0.5 \mathrm{ng} / \mathrm{mL}$ & $>1.2 \mathrm{nmol} / \mathrm{L}$ & 0.989 & 0.943 & $<0.0001$ \\
\hline S & $>0.5 \mathrm{ng} / \mathrm{mL}$ & $>1.0 \mathrm{nmol} / \mathrm{L}$ & 0.986 & 0.920 & $<0.0001$ \\
\hline SS & $>0.5 \mathrm{ng} / \mathrm{mL}$ & $>1.34 \mathrm{nmol} / \mathrm{L}$ & 0.993 & 0.977 & 0.0063 \\
\hline \multicolumn{6}{|c|}{ septic patients versus patients with septic shock patients } \\
\hline & PCT Cut-off & MR-proADM Cut-off & PCT AUCs & MR-proADM AUCs & p $\Delta$ AUCs \\
\hline S vs SS & $>2.7 \mathrm{ng} / \mathrm{mL}$ & $>2.7 \mathrm{nmol} / \mathrm{L}$ & 0.661 & 0.78 & $<0.0001$ \\
\hline \multicolumn{6}{|c|}{ Discriminating cut-off by different microbial etiology in sepsis and septic shock } \\
\hline & PCT Cut-off & MR-proADM Cut-off & PCT AUCs & MR-proADM AUCs & p $\Delta$ AUCs \\
\hline Gram-negative $S$ & $>0.5 \mathrm{ng} / \mathrm{mL}$ & $>1.00 \mathrm{nmol} / \mathrm{L}$ & 0.999 & 0.911 & $<0.0001$ \\
\hline Gram-negative SS & $>0.65 \mathrm{ng} / \mathrm{mL}$ & $>1.34 \mathrm{nmol} / \mathrm{L}$ & 0.999 & 0.979 & 0.0012 \\
\hline Gram-positive S & $>0.3 \mathrm{ng} / \mathrm{mL}$ & $>1.00 \mathrm{nmol} / \mathrm{L}$ & 0.969 & 0.919 & 0.0005 \\
\hline Gram-positive SS & $>0.3 \mathrm{ng} / \mathrm{mL}$ & $>1.50 \mathrm{nmol} / \mathrm{L}$ & 0.977 & 0.972 & n.s \\
\hline Fungal S & $>0.3 \mathrm{ng} / \mathrm{mL}$ & $>1.37 \mathrm{nmol} / \mathrm{L}$ & 0.977 & 0.991 & n.s \\
\hline Fungal SS & $>0.45 \mathrm{ng} / \mathrm{mL}$ & $>1.56 \mathrm{nmol} / \mathrm{L}$ & 0.950 & 0.986 & n.s \\
\hline Polymicrobial S & $>0.42 \mathrm{ng} / \mathrm{mL}$ & $>1.10 \mathrm{nmol} / \mathrm{L}$ & 0.992 & 0.920 & 0.0018 \\
\hline Polymicrobial SS & $>0.53 \mathrm{ng} / \mathrm{mL}$ & $>1.44 \mathrm{nmol} / \mathrm{L}$ & 0.996 & 0.971 & 0.0021 \\
\hline
\end{tabular}

of $S$. aureus and Enterococcus spp. These values were significantly higher than those observed in ConS ( $\mathrm{p}=0.0003$ and $\mathrm{p}=0.0120$ respectively) in agreement with the evidence that among gram-positive isolates, PCT production is more stimulated by S.aureus and Enteroccoccus spp. In case of Streptococcus spp., PCT median values were not statistically different from all other gram-positive isolates. This could suggest that in presence of gram-positive isolates PCT could be produced at similar levels independently from the isolate, except for ConS that presented the lowest levels and $S$. aureus or Enterococcus spp. showing the highest, as previously reported [17-22]. MR-proADM showed homogenous values distribution between different gram-positive isolates, but with significantly higher values in case of septic shock from $S$. aureus, 
A

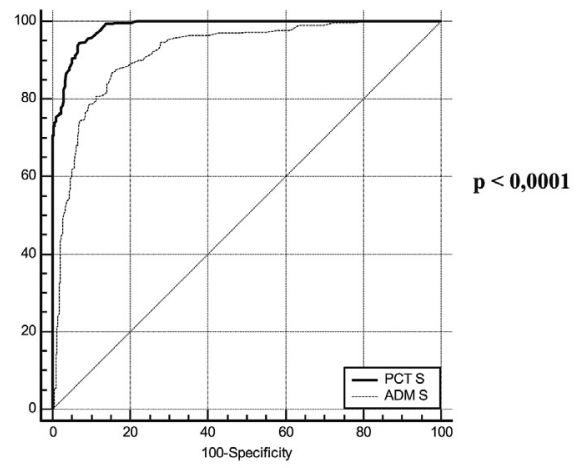

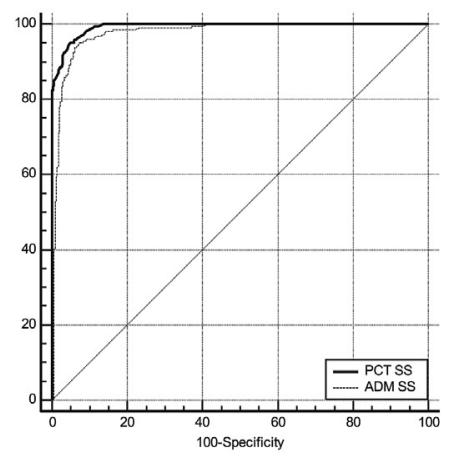

C

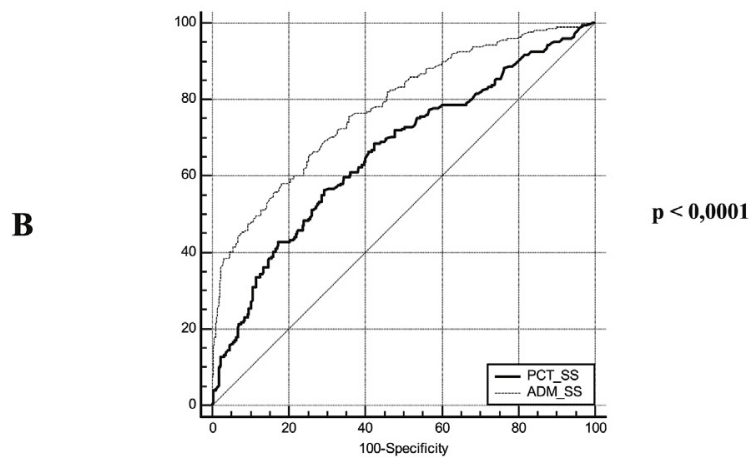

Fig. 5. Roc Curve Analysis. PCT (cut_off $>0.5 \mathrm{ng} / \mathrm{mL}$ ) and MR-proADM (cut-off $>1 \mathrm{nmol} / \mathrm{L}$ ) AUC comparison in septic patients without septic shock diagnosis (S) vs control patients (Panel A); PCT (cut_off $>0.5 \mathrm{ng} / \mathrm{mL}$ ) and MR-proADM (cut-off $>1.3 \mathrm{nmol} / \mathrm{L}$ ) AUC in septic shock patients (SS) vs controls (panel B); PCT (cut_off $>2.7 \mathrm{ng} / \mathrm{mL}$ ) and MR-proADM (cut-off $>2.7 \mathrm{nmol} / \mathrm{L}$ ) AUC comparison in septic shock patients (SS) vs septic patients (Panel C).

Table 5

ROC curve analysis: cut-off values to discriminate gram-negative (GN), Grampositive (GP), Fungal (F) sepsis (S) and septic shock (SS); Area Under Roc curve (AUC), Positive Predictive value (PPV) and Negative Predictive value (NPV).

\begin{tabular}{llllll}
\hline & PCT cut-off & PPV & NPV & PCT AUCs & $\mathrm{p}$ \\
\hline GNvsGP S & $>0.80 \mathrm{ng} / \mathrm{mL}$ & 0.75 & 0.15 & 0.798 & $<0.001$ \\
GNvsGP SS & $>3.88 \mathrm{ng} / \mathrm{mL}$ & 0.89 & 0.52 & 0.785 & $<0.001$ \\
GNvsF S & $>3.57 \mathrm{ng} / \mathrm{mL}$ & 0.96 & 0.81 & 0.804 & $<0.001$ \\
GNvsF SS & $>8.77 \mathrm{ng} / \mathrm{mL}$ & 0.94 & 0.19 & 0.577 & 0.0002 \\
GPvsF S & $>0.56 \mathrm{ng} / \mathrm{mL}$ & 0.28 & 0.12 & 0.600 & 0.034 \\
GPvsF SS & $>1.74 \mathrm{ng} / \mathrm{mL}$ & 0.78 & 0.25 & 0.606 & 0.049 \\
\hline
\end{tabular}

Enterococcus spp. and Streptococcus spp., confirming the prognostic role of MR-proADM in disease progression towards septic shock and organ failure.

In fungal sepsis, PCT may present low values even below the most used cut-off of $0.5 \mathrm{ng} / \mathrm{mL}$, as previously suggested [18-21]. Conversely, the homogeneous distribution of MR-proADM values at rather high levels, especially in case of Candida albicans and Candida tropicalis isolates, suggests that these microorganisms could be typically associated with more severe clinical conditions [44].

In case of polymicrobial etiology, PCT median values were significantly higher when gram-negative isolates were present. This is in agreement with the suggestion that gram-negative isolates generally stimulates PCT release than other microorganisms [20]. Conversely, even if MR-proADM values were higher in presence of gram-negative isolates, any significant difference was found between the various polymicrobial associations being values homogenously distributed.

At ROC curve analysis, PCT and MR-proADM showed very good AUC correctly identifying patients with sepsis and septic shock. Cut-off values to be considered at the onset of the clinical signs and symptoms have been identified and analyzed in relationship with the microbial etiology.

Discriminating PCT cut-off values for gram-negative versus grampositive or fungal sepsis were analyzed stratifying by sepsis or septic shock. The highest values were identified in case of septic shock. In previous studies [17-22] discriminating cut-off values were not analyzed by this stratification, but the stratification used in our study allows to better characterized cut-offs needed for best diagnostic accuracy. The results showed that the highest PPV was associated with the cut-off discriminating gram-negative from fungal sepsis and septic shock $(>3.57 \mathrm{ng} / \mathrm{mL}$, PPV 0.96 and $>8.77 \mathrm{ng} / \mathrm{mL}$, PPV 0.96, respectively). A good diagnostic accuracy was also evidenced to discriminate gram-negative from gram-positive septic shock (>3.88 ng/ $\mathrm{mL}$ PPV 0.89). Lower diagnostic accuracy were evidenced to discriminate gram-negative and gram-positive sepsis $(>0.80 \mathrm{ng} / \mathrm{mL}$, PPV 0.78 ) and gram-positive from fungal septic shock ( $>1.74 \mathrm{ng} / \mathrm{mL}$ PPV 0.75), whereas the lowest PPV (0.28) was evidenced to distinguish gram-positive from fungal sepsis. These results are in line with other studies [19-22] and confirm the ability of PCT to discriminate gramnegative from gram-positive and fungal etiologies, especially in case of septic shock. The etiological diagnostic role of PCT represents an advantage to provide early tailored antimicrobial therapy since the first hours of the septic event. PCT accuracy was lower in gram-positive and fungal sepsis discrimination, because of the lower PCT ability to identify these etiologies, as previously reported [19-22]. The cut-off proposed are slightly different from other studies, but in this study cut-off values have been estimated on PCT measurement performed at the same moment of blood culture collection (first sign of sepsis or septic shock) and stratifying patients by sepsis and septic shock. This specific way to determine cut-off has not been previously used and it is the most appropriate to provide values to be used at the first suspicious of sepsis or septic shock.

MR-proADM discriminating cut-offs were homogeneously distributed in Gram-negative and Gram-positive sepsis and higher in septic shock. These data are in agreement with the prognostic role of this biomarker [11-15,23,24,26-29], in fact values are not influenced by the different pathogen etiologies but are increased in case of disease severity progression and in case of fungal sepsis that generally underline severe clinical conditions. MR-proADM combination with PCT 

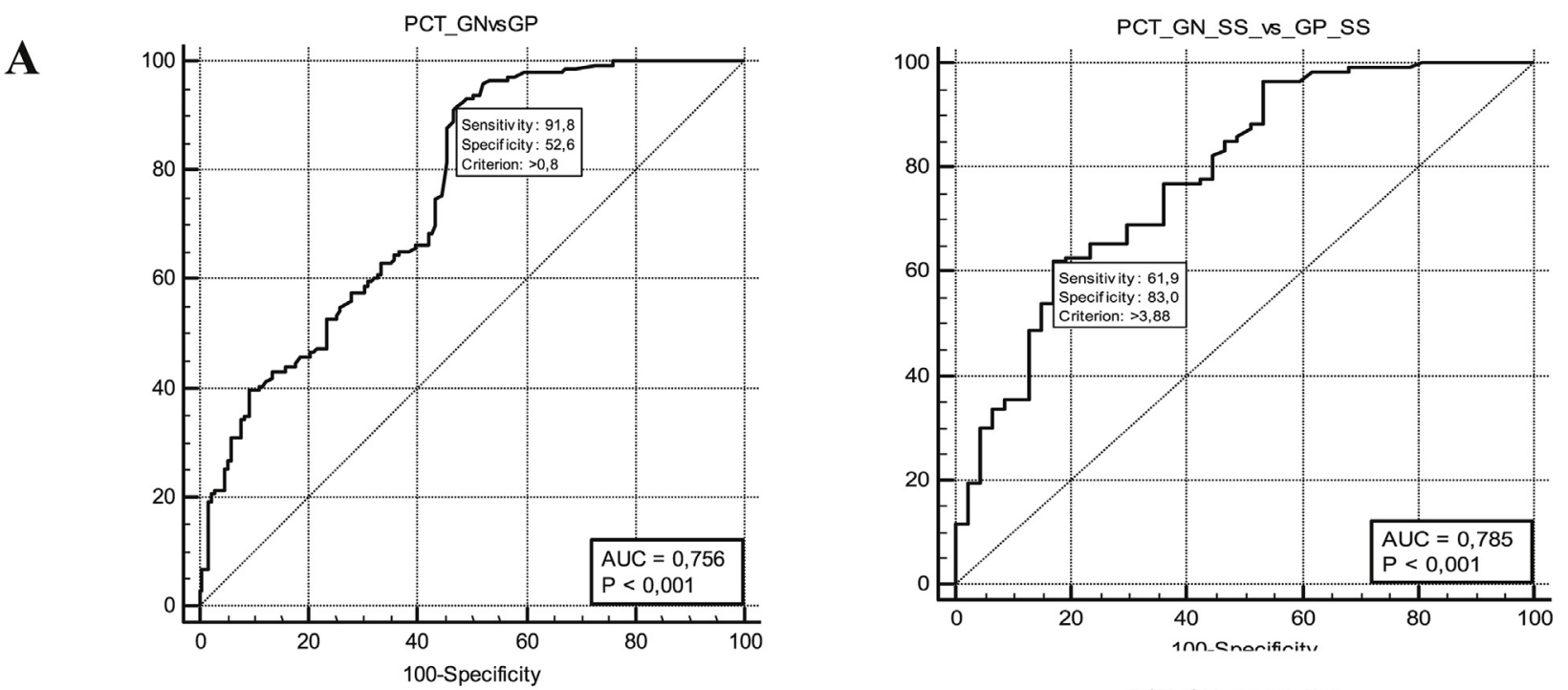

B
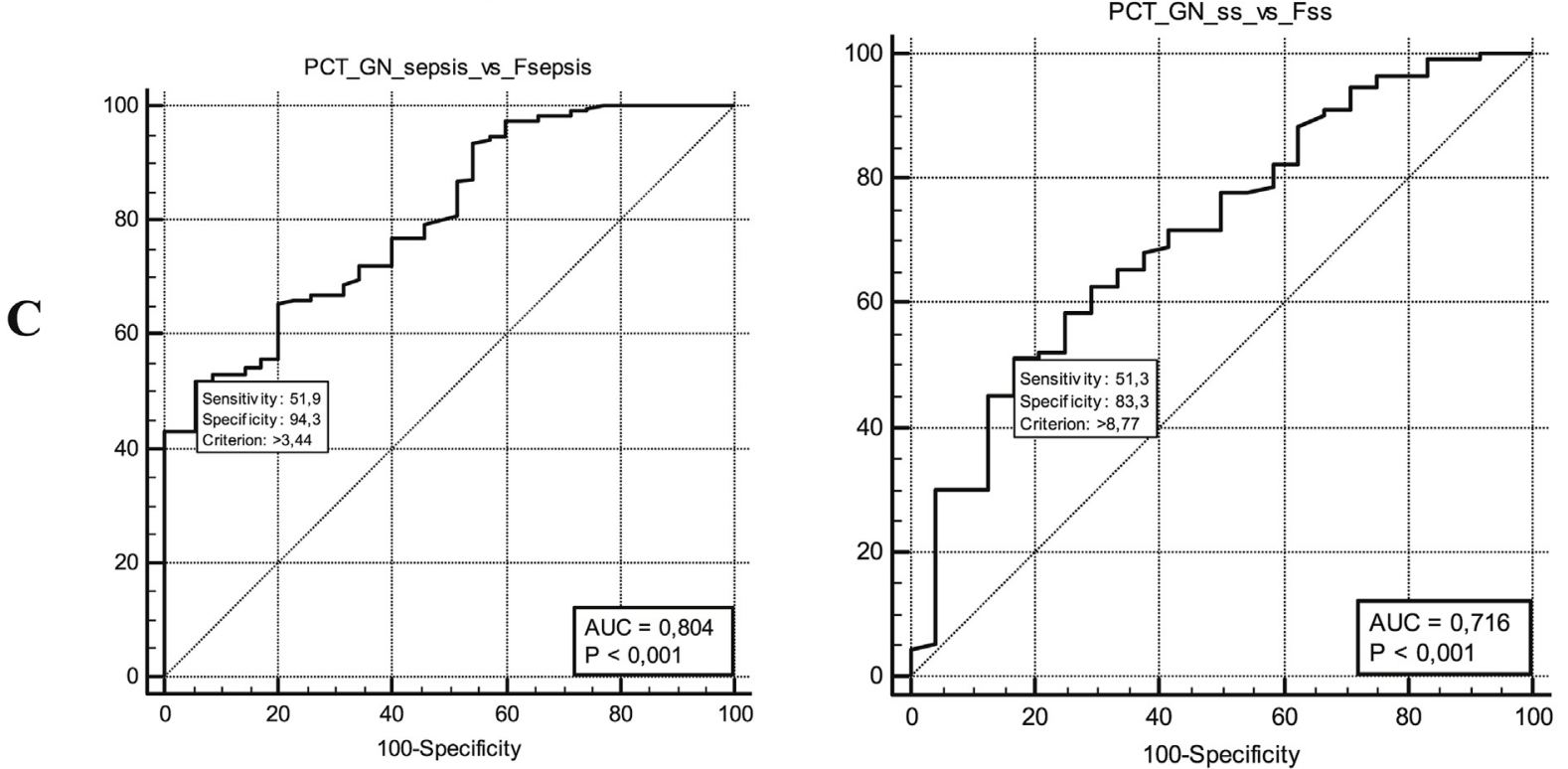

Fig. 6. ROC curve analysis to differentiate gram-negative sepsis from gram-positive sepsis stratifying for sepsis and SS. Area Under the Curve (AUC).
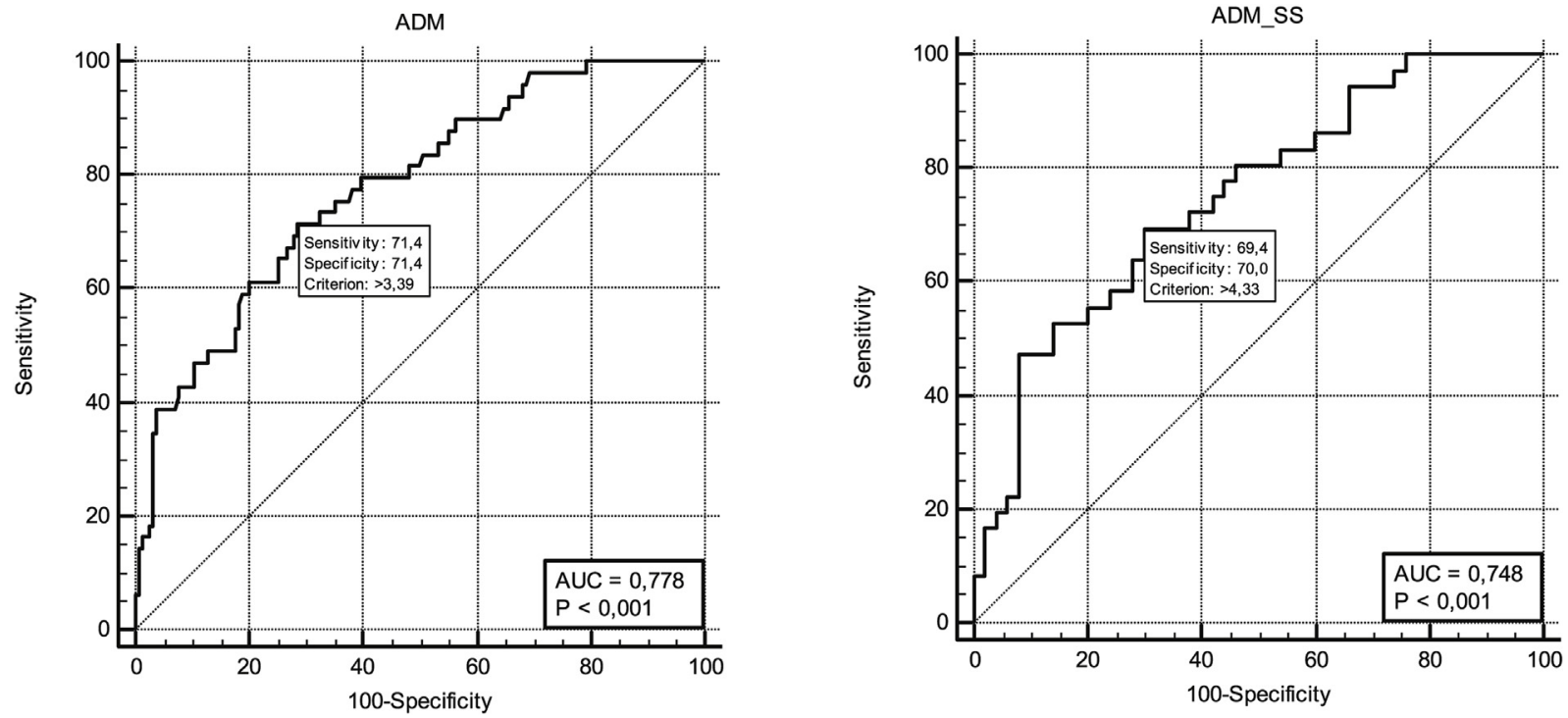

Fig. 7. ROC curve analysis to differentiate fungal sepsis from gram-positive sepsis stratifying for sepsis and SS. Area Under the Curve (AUC). 

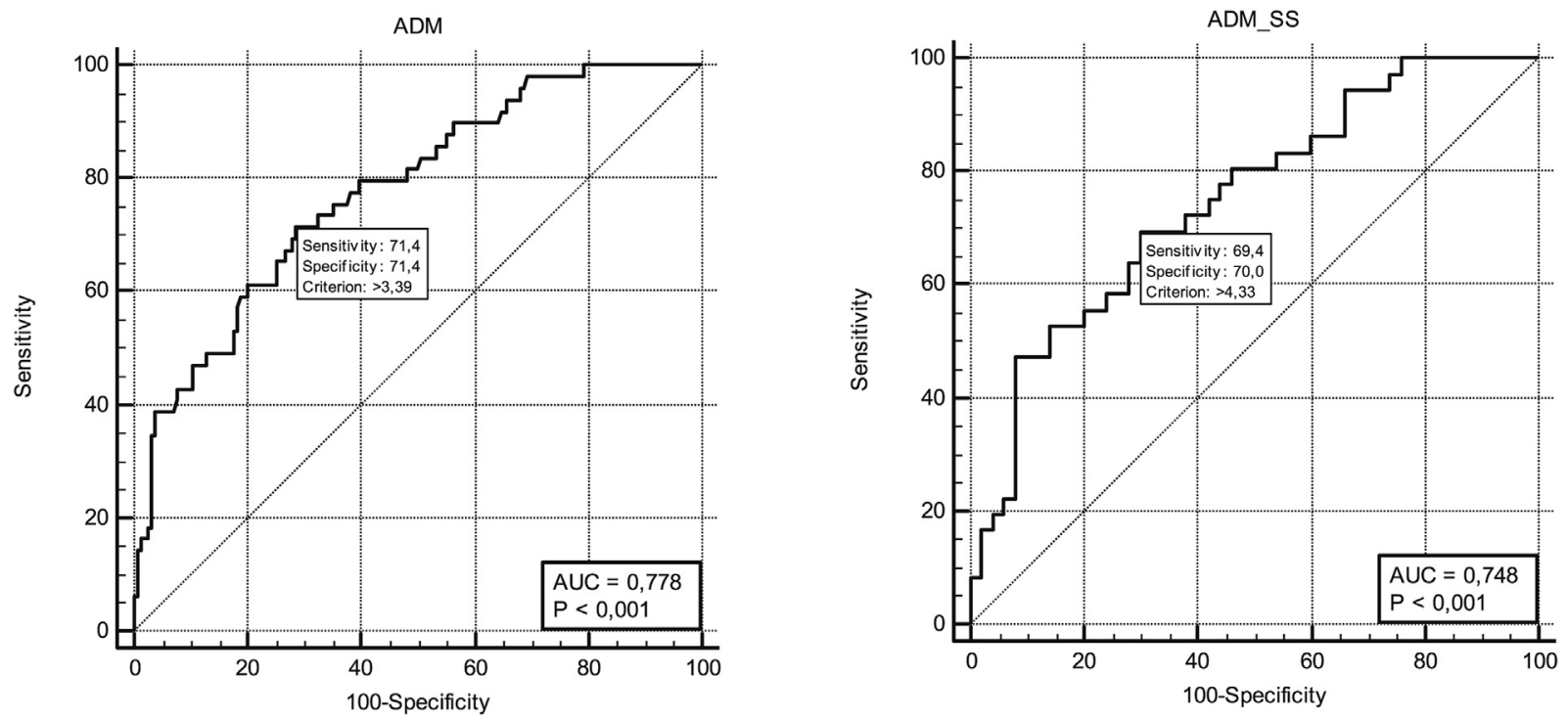

Fig. 8. MR-proADM and 90-days mortality in sepsis (S) and septic shock (SS).

could aid to predict sepsis patient progression towards septic shock and multi-organ failure. Cut-off values $>3.39 \mathrm{nmol} / \mathrm{L}$ in sepsis and $>$ $4.33 \mathrm{nmol} / \mathrm{L}$ in septic shock have been associated with significant higher risk of 90-days mortality, thus confirming the prognostic role of the biomarker.

\section{Conclusion}

The combination of different cut-off values of both markers could aid to predict the etiology as well as the severity of sepsis. At our knowledge, this is the first study where the combination of different biomarkers has been used to determine the diagnostic PCT cut-off levels based on the different microbial etiology and the prognostic MRproADM levels in sepsis and septic shock. Data from the study showed that PCT and MR-proADM combination represents an advantage for sepsis diagnosis and prognosis to provide early tailored therapy and risk stratification for 90-days mortality.

\section{Ethical issues}

We certify that all data collected during the study is reported in this manuscript and no data from the study has been published or will be published elsewhere.

\section{Authors' contribution and article approval}

All authors were involved in study design, data collection and manuscript preparation.

\section{Declaration of competing interest}

The authors declare that they have no competing interests.

\section{References}

[1] M. Shankar-Hari, G.S. Phillips, M.L. Levy, C.W. Seymour, V.X. Liu, C.S. Deutschman, et al., Developing a new definition and assessing new clinical criteria for septic shock: for the Third international Consensus definitions for sepsis and septic shock (Sepsis-3), JAMA 315 (8) (2016) 775-787.

[2] M. Singer, C.S. Deutschman, C.W. Seymour, M. Shankar-Hari, D. Annane, M. Bauer, et al., The Third international Consensus definitions for sepsis and septic shock (Sepsis-3), JAMA 315 (8) (2016) 801-810.

[3] J. Cohen, J.L. Vincent, N.K. Adhikari, F.R. Machado, D.C. Angus, T. Calandra, et al., Sepsis: a roadmap for future research, Lancet Infect. Dis. 15 (5) (2015) 581-614.

[4] J. Garnacho-Montero, A. Gutiérrez-Pizarraya, A. Escoresca-Ortega, E. Fernández-
Delgado, J.M. López-Sánchez, Adequate antibiotic therapy prior to ICU admission in patients with severe sepsis and septic shock reduces hospital mortality, Crit. Care 19 (2015) 302.

[5] G. Vazquez-Grande, A. Kumar, Optimizing antimicrobial therapy of sepsis and septic shock: focus on antibiotic combination therapy, Semin. Respir. Crit. Care Med. 36 (1) (2015) 154-166.

[6] R.P. Peters, M.A. van Agtmael, S.A. Danner, P.H. Savelkoul, C.M. VandenbrouckeGrauls, New developments in the diagnosis of bloodstream infections, Lancet Infect. Dis. 4 (12) (2004) 751-760.

[7] D. Fontanals, I. Sanfeliu, I. Pons, D. Mariscal, M. Torra, Evaluation of the BacT/alert and VITAL blood culture systems for the diagnosis of bacteremia, Clin. Microbiol. Infect. 4 (2) (1998) 88-93.

[8] A. Lee, S. Mirrett, L.B. Reller, M.P. Weinstein, Detection of bloodstream infections in adults: howmany blood cultures are needed? J. Clin. Microbiol. 45 (11) (2007) 3546-3548.

[9] C. Pierrakos, J.L. Vincent, Sepsis biomarkers: a review, Crit. Care 14 (1) (2010) R15.

[10] D.K. Matthaiou, G. Ntani, M. Kontogiorgi, G. Poulakou, A. Armaganidis, G. Dimopoulos, An ESICM systematic review and meta-analysis of procalcitonin guided antibiotic therapy algorithms in adult critically ill patients, Intensive Care Med. 38 (6) (2012) 940-949.

[11] S. Angeletti, F. Battistoni, M. Fioravanti, S. Bernardini, G. Dicuonzo, Procalcitonin and mid-regional pro-adrenomedullin test combination in sepsis diagnosis, Clin. Chem. Lab. Med. 51 (5) (2013) 1059-1067.

[12] S. Angeletti, S. Spoto, M. Fogolari, M. Cortigiani, M. Fioravanti, et al., Diagnostic and prognostic role of procalcitonin (PCT) and MR-pro- Adrenomedullin (MRproADM) in bacterial infections, APMIS 123 (9) (2015) 740-748.

[13] S. Angeletti, G. Dicuonzo, M. Fioravanti, M. De Cesaris, M. Fogolari, A. Lo Presti, M. Ciccozzi, L De Florio Procalcitonin, MR-proadrenomedullin, and cytokines measurement in sepsis diagnosis: advantages from test combination, Dis. Markers 2015 (2015) 951532.

[14] S. Angeletti, M. Ciccozzi, M. Fogolari, S. Spoto, A. Lo Presti, S. Costantino, G. Dicuonzo, Procalcitonin and MR-proAdrenomedullin combined score in the diagnosis and prognosis of systemic and localized bacterial infections, J. Infect. 72 (3) (2016) 395-398.

[15] S. Spoto, E. Cella, M. De Cesaris, L. Locorriere, S. Mazzaroppi, E. Nobile, et al., Procalcitonin and MR-Proadrenomedullin combination with SOFA and qSOFA scores for sepsis diagnosis and prognosis: a diagnostic algorithm, Shock 50 (1) (2018) 44-52 2018.

[16] F. Valenzuela-Sánchez, B. Valenzuela-Méndez, J.F. Rodríguez-Gutiérrez, A. EstellaGarcía, M.A. González-García, New role of biomarkers: mid-regional-pro-adrenomedullin: the biomarker of organ failure, Ann. Transl. Med. 4 (17) (2016) 329.

[17] C. He, B. Wang, Y.F. Wang, Y.C. Shen, Can procalcitonin be used to diagnose Gramnegative bloodstream infection? Evidence based on a meta-analysis, Eur. Rev. Med. Pharmacol. Sci. 21 (14) (2017) 3253-3261.

[18] S.Y. Guo, Y. Zhou, Q.F. Hu, J. Yao, H. Wang, Procalcitonin is a marker of gramnegative bacteremia in patients with sepsis, Am. J. Med. Sci. 349 (6) (2015) 499-504.

[19] D.O. Thomas-Rüddel, B. Poidinger, M. Kott, M. Weiss, K. Reinhart, F. Bloos, Influence of pathogen and focus of infection on procalcitonin values in sepsis patients with bacteremia or candidemia, Crit. Care 22 (1) (2018) 128.

[20] C. Leli, M. Ferranti, A. Moretti, Z.S. Al Dhahab, E. Cenci, A. Mencacci, Procalcitonin levels in gram-positive, gram-negative, and fungal bloodstream infections, Dis. Markers 2015 (2015) 701480.

[21] T. Arai, S. Ohta, J. Tsurukiri, K. Kumasaka, K. Nagata, T. Okita, et al., Procalcitonin levels predict to identify bacterial strains in blood cultures of septic patients, Am. J. Emerg. Med. 34 (11) (2016) 2150-2153. 
[22] S. Li, H. Rong, Q. Guo, Y. Chen, G. Zhang, J. Yang, Serum procalcitonin levels distinguish Gram-negative bacterial sepsis from Gram-positive bacterial and fungal sepsis, J. Res. Med. Sci. 21 (2016) 39.

[23] B. Viaggi, D. Poole, O. Tujjar, S. Marchiani, A. Ognibene, S. Finazzi, Mid regional pro-adrenomedullin for the prediction of organ failure in infection. Results from a single centre study, PLoS One 13 (8) (2018) e0201491.

[24] G. Elke, F. Bloos, D.C. Wilson, F.M. Brunkhorst, J. Briegel, K. Reinhart, et al., The use of mid-regional proadrenomedullin to identify disease severity and treatment response to sepsis - a secondary analysis of a large randomised controlled trial, Crit. Care 22 (1) (2018) 79.

[25] J.M. Legramante, M. Mastropasqua, B. Susi, O. Porzio, M. Mazza, G. Miranda Agrippino, C. D' Agostini, et al., Prognostic performance of MR-pro-adrenomedullin in patients with communityacquired pneumonia in the Emergency Department compared to clinical severity scores PSI and CURB, PLoS One 12 (11) (2017) $\mathrm{e} 0187702$.

[26] P.E. Charles, E. Péju, A. Dantec, R. Bruyère, N. Meunier-Beillard, A. Dargent, et al., Mr-proadm elevation upon icu admission predicts the outcome of septic patients and is correlated with upcoming fluid overload, Shock 48 (4) (2017) 418-426.

[27] J. Ni, Y. Sun, H. Qu, A. Wang, Y. Cao, X. Li, Prognostic value of serum proadrenomedullin in catheter-related bloodstream infection in the intensive care unit: a prospective observational study, Medicine (Baltim.) 97 (42) (2018) e12821.

[28] F. Valenzuela-Sánchez, B. Valenzuela-Méndez, R.B. Austria, J.F. RodríguezGutiérrez, A. Estella García, L. Fernández-Ruiz, et al., Plasma levels of mid-regional pro-adrenomedullin in sepsis are associated with risk of death, Minerva Anestesiol. 85 (4) (2019) 366-375.

[29] G. Elke, F. Bloos, D.C. Wilson, F.M. Brunkhorst, J. Briegel, K. Reinhart, et al., The use of mid-regional proadrenomedullin to identify disease severity and treatment response to sepsis - a secondary analysis of a large randomised controlled trial, Crit. Care 22 (1) (2018) 79.

[30] M.M. Levy, M.P. Fink, J.C. Marshall, E. Abraham, D. Angus, D. Cook, et al., 2001 SCCM/ESICM/ACCP/ATS/SIS. International sepsis definitions conference, Crit. Care Med. 31 (4) (2003) 1250-1256.

[31] A. Albert, On the use and computation of likelihood ratios in clinical chemistry, Clin. Chem. 28 (5) (1982) 1113-1119.

[32] M. Oberhoffer, I. Stonans, S. Russwurm, E. Stonane, H. Vogelsang, U. Junker, et al., Procalcitonin expression in human peripheral blood mononuclear cells and its modulation by lipopolysaccharides and sepsis-related cytokines in vitro, J. Lab.
Clin. Med. 134 (1) (1999) 49-55.

[33] S. Li, H. Rong, Q. Guo, Y. Chen, G. Zhang, J. Yang, Serum procalcitonin levels distinguish Gram-negative bacterial sepsis from Gram-positive bacterial and fungal sepsis, J. Res. Med. Sci. 21 (2016) 39.

[34] O. Beran, R. Potmešil, M. Holub, Differences in Toll-like receptor expression and cytokine production after stimulation with heat-killed gram-positive and gram-negative bacteria, Folia Microbiol. 56 (2011) 138-142.

[35] E. Tavares, R. Maldonado, M.L. Ojeda, F.J. Miñano, Circulating inflammatory mediators during start of fever in differential diagnosis of gram-negative and grampositive infections in leukopenic rats, Clin. Diagn. Lab. Immunol. 12 (2005) 1085-1093.

[36] A. Plato, S.E. Hardison, G.D. Brown, Pattern recognition receptors in antifungal immunity, Semin. Immunopathol. 37 (2) (2015) 97-106.

[37] S. Vautier, D.M. MacCallum, G.D. Brown, C-type lectin receptors and cytokines in fungal immunity, Cytokine 58 (1) (2012) 89-99.

[38] S.J. Park, M.A. Hughes, M. Burdick, R.M. Strieter, B. Mehrad, Early NK cell-derived IFN-\{gamma\} is essential to host defense in neutropenic invasive aspergillosis, J. Immunol. 182 (7) (2009) 4306-4312.

[39] P. Linscheid, D. Seboek, E.S. Nylen, I. Langer, M. Schlatter, K.L. Becker, et al., In vitro and in vivo calcitonin I gene expression in parenchymal cells: a novel product of human adipose tissue, Endocrinology 144 (12) (2003) 5578-5584.

[40] Y.H. Dou, J.K. Du, H.L. Liu, X.D. Shong, The role of procalcitonin in the identification of invasive fungal infection-a systemic review and meta-analysis, Diagn. Microbiol. Infect. Dis. 76 (4) (2013) 464-469.

[41] Y. Fu, J. Chen, B. Cai, J. Zhang, L. Li, C. Liu, et al., The use of PCT, CRP, IL-6 and SAA in critically ill patients for an early distinction between candidemia and Gram positive/negative bacteremia, J. Infect. 64 (4) (2012) 438-440.

[42] A. Martini, L. Gottin, N. Menestrina, V. Schweiger, D. Simion, J.L., Vincent Procalcitonin levels in surgical patients at risk of candidemia, J. Infect. 60 (6) (2010) 425-430.

[43] J.E. Stalenhoef, C. van Nieuwkoop, D.C. Wilson, W.E. van der Starre, N.M. Delfos, E.M.S. Leyten, et al., Biomarker guided triage can reduce hospitalization rate in community acquired febrile urinary tract infection, J. Infect. 77 (1) (2018) 18-24.

[44] R. Khatib, L.B. Johnson, M.G. Fakih, K. Riederer, L. Briski, Current trends in candidemia and species distribution among adults: Candida glabrata surpasses C. albicans in diabetic patients and abdominal sources, Mycoses 59 (12) (2016) $781-786$. 